\title{
Extremal Solutions for a Class of Unilateral Problems
}

\author{
N. B. Huy, N. D. Thanh and T. D. Thanh
}

\begin{abstract}
We apply a fixed point theorem for increasing operators in ordered Banach spaces to prove the existence of extremal (i.e. maximal or minimal) solutions for the variational inequality $\langle A v, w-v\rangle \geq \int_{\Omega} f(x, v)(w-v) d x$ where $A$ is the $p$ Laplacian and $f(x, u)=F(x, u, u)$ with $F(x, u, v)$ being a function, non-decreasing in $u$ and non-increasing in $v$.

Keywords: Fixed points, increasing operators, ordered spaces, extremal solutions, variational inequalities, unilateral problems
\end{abstract}

AMS subject classification: 49J85, 47H07

\section{Introduction}

Fixed point theorems for monotone increasing operators in ordered spaces are an useful tool for studying elliptic and parabolic differential equations. They allow us to investigate the existence and approximation of solutions and extremal (i.e. maximal or minimal) solutions for both continuous and discontinuous equations (see $[1,3,5,7]$ and the references therein). In the present paper we shall apply such a theorem to prove the existence of extremal solutions for a class of differential variational inequalities. To our knowledge, the approach considered has received little attention in studying inequalities.

We will study the existence of extremal solutions for the variational inequality to find $v$ such that

$$
\left\{\begin{array}{l}
v \in K, f(x, v), v f(x, v) \in L^{1}(\Omega) \\
\langle A v, w-v\rangle \geq \int_{\Omega} f(x, v)(w-v) d x \quad \forall w \in K \cap L^{\infty}(\Omega) .
\end{array}\right.
$$

Nguyen Bich Huy and Nguyen Duy Thanh: Coll. Edu., Dept. Math., 280 An Duong Vuong, Ho Chi Minh City, Viet Nam; nguyenbichhuy@hcm.vnn.vn

Tran Dinh Thanh: Coll. Medicine \& Pharmacy, Dept. Math., 217 An Duong Vuong, Ho Chi Minh City, Viet Nam

ISSN 0232-2064 / \$2.50 C Heldermann Verlag Berlin 
Here,

$\Omega$ is an open bounded domain in $\mathbb{R}^{N}$ with smooth boundary

$A v=-\operatorname{div}\left(|\nabla v|^{p-2} \nabla v\right)$ is the $p$-Laplacian with $1<p<N$

$K=\left\{w \in W_{0}^{1, p}(\Omega): w \geq \varphi\right.$ a.e. in $\left.\Omega\right\}$ with $0 \leq \varphi \in W_{0}^{1, p}(\Omega) \cap L^{\infty}(\Omega)$

$\langle\cdot, \cdot\rangle$ stands for the dual pairing between $W^{1, p}(\Omega)$ and $W^{-1, p^{\prime}}(\Omega)$.

On the function $f$ besides suitable growth conditions we will impose the monotonocity assumption $f(x, u)=F(x, u, u)$ where $F(x, u, v)$ is a function, non-decreasing in $u$ and non-increasing in $v$. This assumption allows us to reduce variational inequality (1) to a problem of finding fixed points for monotone increasing operators in ordered spaces.

Our paper is related with the recent papers [8, 9] of Vy Khoi Le, in which a general notion of subsolutions and supersolutions for variational inequalities has been defined and some interesting existence results of extremal solutions are given. In comparison with those papers, we use a different method based on reducing inequality (1) to an operator equation and allowing non-linearities in our consideration to be discontinuous. Our results are also related with the paper [4] of Drabek and Hernandez, which deals with a similar problem but in the setting of equations.

\section{Preparatory results}

2.1 A fixed point theorem in ordered spaces. Let $X$ be a real Banach space and $C \subset X$ be a cone, i.e. $C$ is a closed convex subset such that $t C \subset C$ for all $t \geq 0$ and $C \cap(-C)=\{0\}$. We define a partial ordering $\leq$ with respect to $C$ by $x \leq y$ if $y-x \in C$. We will write $[u, v]=\{x \in X: u \leq x \leq v\}$ if $u \leq v$ and $[u, \infty)=\{x \in X: u \leq x\}$. We say that a set $M \subset X$ is directed up-wards if for all $u, v \in M$ there exists $w \in M$ such that $u, v \leq w$. A map $T: M \subset X \rightarrow X$ is called increasing if $x, y \in M$ with $x \leq y$ implies $T x \leq T y$.

Combining [5: Theorem 1.2.1] and [7: Theorem 1] we obtain the following

Theorem A. Let $X$ be a Banach space ordered by a cone $C, M \subset X$ be a closed subset and $T: M \rightarrow M$ be an increasing operator such that:

(i) The set $M_{0}=\{u \in M: u \leq T u\}$ is non-empty and directed up-wards.

(ii) The sequence $\left\{T u_{n}\right\}$ converges whenever $\left\{u_{n}\right\}$ is increasing in $M_{0}$. Then for each element $u \in M_{0}$ the operator $T$ has a maximal fixed point $u^{*}$ and a minimal fixed point $u_{*}$ in $M \cap[u, \infty)$ in the sense that if $\bar{u}$ is a fixed point of $T$ in $M \cap[0, \infty)$, then $u_{*} \leq \bar{u} \leq u^{*}$.

Remark. In our applications of Theorem $\mathrm{A}$ in Section $3, X$ will be a Lebesgue space $L^{p_{0}}(\Omega)$ with the cone $C$ of non-negative functions. In this case, to prove convergence of an increasing sequence $\left\{T u_{n}\right\}$ it is sufficient to verify its boundedness. 
2.2 Reduction of inequality (1) to an operator equation. Let us recall that for $1<p<N$ we have the continuous imbedding $W^{1, p}(\Omega) \hookrightarrow$ $L^{p^{*}}(\Omega)$ where $p^{*}=\frac{N p}{N-p}$ and hence, we obtain the continuous imbedding $L^{\left(p^{*}\right)^{\prime}}(\Omega) \hookrightarrow W^{-1, p^{\prime}}(\Omega)$ by duality. In $W_{0}^{1, p}(\Omega)$ we use the usual norm $\|u\|_{1, p}=\left(\int_{\Omega}|\nabla u|^{p} d x\right)^{1 / p}$ and we denote by $\|x\|_{t}$ the norm in the Lebesgue space $L^{t}(\Omega)$.

In reducing variational inequality (1) to an operator equation we need the following auxiliary results from [2].

Theorem B. Let $z \in W^{-1, p}(\Omega)$ and $g: \Omega \times \mathbb{R} \rightarrow \mathbb{R}$ be a Carathéodory function such that

$$
\begin{aligned}
& g(x, u) \text { is non-decreasing in } u \text { and } g(x, 0)=0 \\
& \sup \{|g(x, u)|:|u| \leq t\}=h_{t}(x) \in L^{1}(\Omega) \text { for all } t \geq 0 .
\end{aligned}
$$

Then the variational inequality

$$
\left\{\begin{array}{l}
v \in K, g(x, v), v g(x, v) \in L^{1}(\Omega) \\
\langle A v-z, w-v\rangle+\int_{\Omega} g(x, v)(w-v) d x \geq 0 \quad \forall w \in K \cap L^{\infty}(\Omega)
\end{array}\right.
$$

has a unique solution $v$ satisfying

$$
\langle A v-z, \varphi-v\rangle+\int_{\Omega} g(x, v)(\varphi-v) d x=0 .
$$

Theorem C. Let $u \in W_{0}^{1, p}(\Omega)$ and $z \in W^{-1, p^{\prime}}(\Omega)$ such that $u \geq 0$ a.e. in $\Omega$ and $z=\mu+h$ where $\mu$ is a positive Radon measure and $h \in L^{1}(\Omega)$. Moreover, assume that $h u \geq v$ a.e. in $\Omega$ for some $v \in L^{1}(\Omega)$. Then $h u$ belongs to $L^{1}(\Omega), u$ (or more exactly, the quasicontinuous representative of $u$ ) belongs to $L^{1}(\Omega, d \mu)$ and

$$
\langle\mu+h, u\rangle=\int_{\Omega} u d \mu+\int_{\Omega} h u d x \geq \int_{\Omega} h u d x .
$$

Now, we return to variational inequality (1). On the function $f=f(x, u)$ we shall make the hypotheses $f(x, u)=F(x, u, u)$ where $F: \Omega \times \mathbb{R}^{+} \times \mathbb{R}^{+} \rightarrow \mathbb{R}$ satisfies:

$$
\left\{\begin{array}{l}
(x, u) \mapsto F(x, u, v) \text { is measurable for all } v \in \mathbb{R}^{+} \\
v \mapsto F(x, u, v) \text { is continuous for all } u \in \mathbb{R}^{+} \text {and for a.e. } x \in \Omega .
\end{array}\right.
$$

(H2) $\left\{\begin{array}{l}F(x, 0,0)=0 \text { for a.e. } x \in \Omega \\ u \mapsto F(x, u, v) \text { is non-decreasing for all }(x, v) \in \Omega \times \mathbb{R}^{+} \\ v \mapsto F(x, u, v) \text { is non-increasing for all }(x, u) \in \Omega \times \mathbb{R}^{+} .\end{array}\right.$ 
(H3) $F(x, 0, v) \in L^{1}(\Omega)$ for all $v>0$ and there exist numbers $a, q, r>0$ and a function $m \in L^{s}(\Omega)$ such that

$$
\begin{aligned}
F(x, u, 0)-F(x, u, v) & \geq a v^{q} \\
F(x, u, 0) & \leq m(x) u^{r}
\end{aligned}
$$

and $r<q$.

(H4) At least one of the conditions

$$
\begin{aligned}
& \left(p^{*}\right)^{\prime} \leq \frac{s p^{*}}{s r+p^{*}} \\
& \left(p^{*}\right)^{\prime} \leq \frac{s(q+1)}{s r+q+1}
\end{aligned}
$$

holds.

In the sequel, we put $p_{0}=p^{*}$ or $p_{0}=q+1$ according to whether condition (4) or (5) holds.

Lemma 1. Let Hypotheses (H1) - (H4) be satisfied. Then for each function $u \in[\varphi, \infty) \subset L^{p_{0}}(\Omega)$ the variatioanal inequality

$$
\left\{\begin{array}{l}
v \in K, F(x, u, v), v F(x, u, v) \in L^{1}(\Omega) \\
\langle A v, w-v\rangle \geq \int_{\Omega} F(x, u, v)(w-v) d x \quad \forall w \in K \cap L^{\infty}(\Omega)
\end{array}\right.
$$

has a unique solution $v$ satisfying

$$
\langle A v, \varphi-v\rangle=\int_{\Omega} F(x, u, v)(\varphi-v) d x .
$$

Proof. Since $u \in L^{p_{0}}(\Omega)$ and $m \in L^{s}(\Omega)$, we have $m(x) u^{r} \in L^{t}(\Omega)$ where $t=\frac{s p_{0}}{s r+p_{0}}$. Therefore, $F(x, u, 0) \in L^{\left(p^{*}\right)^{\prime}}$ by (3) and Hypothesis (H4), and so $F(x, u, 0) \in W^{-1, p^{\prime}}(\Omega)$. Hence, we can rewrite inequality (6) as

$$
\langle A v-F(x, u, 0), w-v\rangle+\int_{\Omega}(F(x, u, 0)-F(x, u, v))(w-v) d x \geq 0 .
$$

The function $g(x, v)=F(x, u, 0)-F(x, u, v)$, extended for $v \in(-\infty, 0)$ by putting $g(x, v)=-g(x,-v)$, is non-decreasing in $v$ and satisfies

$$
\sup \{|g(x, v)|:|v| \leq t \mid\} \leq F(x, u, 0)-F(x, 0, t) \in L^{1}(\Omega) .
$$

Consequently, by Theorem B inequality (8) has a unique solution $v$ satisfying (7) and

$$
\left.\begin{array}{r}
F(x, u, 0)-F(x, u, v) \\
v(F(x, u, 0)-F(x, u, v))
\end{array}\right\} \in L^{1}(\Omega) .
$$

Since $v \in L^{p^{*}}(\Omega)$ and $F(x, u, 0) \in L^{\left(p^{*}\right)^{\prime}}(\Omega)$, from (9) we have that $F(x, u, v) \in$ $L^{1}(\Omega)$ as well as $v F(x, u, v) \in L^{1}(\Omega)$ and hence, $v$ is the unique solution of inequality (6). The proof is complete 
Definition (see $[8,9])$. A function $u_{0} \in W^{1, p}(\Omega)$ is called a $W$-supersolution of variational inequality (1) if $u_{0} \geq 0$ on $\partial \Omega, f\left(x, u_{0}\right) \in L^{\left(p^{*}\right)^{\prime}}(\Omega)$ and

$$
\left\langle A u_{0}, w-u_{0}\right\rangle \geq \int_{\Omega} f\left(x, u_{0}\right)\left(w-u_{0}\right) d x
$$

for all $w$ of the form $w=\max \left\{u_{0}, v\right\}$ with some $v \in K$.

Lemma 2. Let $T$ be the operator that assigns to each $u \in[\varphi, \infty) \subset$ $L^{p_{0}}(\Omega)$ the unique solution of inequality $(6)$. Then $T$ is acting from $[\varphi, \infty) \subset$ $L^{p_{0}}(\Omega)$ into itself and has the following properties:

1) $T$ is increasing.

2) The set $\{u: \varphi \leq u \leq T u\}$ is directed up-wards.

3) If $u_{0}$ is a $W$-supersolution of inequality (1), then $T u_{0} \leq u_{0}$.

Proof. We have $\varphi \leq T u \in W_{0}^{1, p}(\Omega)$, hence $T u \in L^{p_{0}}(\Omega)$ if $p_{0}=p^{*}$. In the case $p_{0}=q+1$ we get from (2) and (9) that $(T u)^{q+1} \in L^{1}(\Omega)$ and so $T u \in L^{p_{0}}(\Omega)$. Thus, $T$ is acting from $[\varphi, \infty) \subset L^{p_{0}}(\Omega)$ into itself.

1) Given $u_{1}, u_{2} \in[\varphi, \infty)$ with $u_{1} \leq u_{2}$ we set $v_{1}=T u_{1}$ and $v_{2}=T u_{2}$, further $\Omega_{1}=\left\{x \in \Omega: v_{1}(x) \leq v_{2}(x)\right\}$ and $\Omega_{2}=\Omega \backslash \Omega_{1}$, and shall prove that

$$
\begin{aligned}
& \left\langle A v_{1}, v_{1}-\left(v_{1}-v_{2}\right)^{+}-\varphi\right\rangle \geq \int_{\Omega} F\left(x, u_{1}, v_{1}\right)\left(v_{1}-\left(v_{1}-v_{2}\right)^{+}-\varphi\right) d x(11) \\
& \left\langle A v_{2}, v_{2}-\left(v_{1}-v_{2}\right)^{+}-\varphi\right\rangle \geq \int_{\Omega} F\left(x, u_{2}, v_{2}\right)\left(v_{2}+\left(v_{1}-v_{2}\right)^{+}-\varphi\right) d x .
\end{aligned}
$$

To show (11), we will apply Theorem $\mathrm{C}$ with

$$
\begin{aligned}
& u=v_{1}-\left(v_{1}-v_{2}\right)^{+}-\varphi \\
& \mu=A v_{1}-F\left(x, u_{1}, v_{1}\right) \\
& h=F\left(x, u_{1}, v_{1}\right) .
\end{aligned}
$$

All the hypotheses of Theorem C are fulfilled since $u=\min \left\{v_{1}, v_{2}\right\}-\varphi \geq 0$, $\mu$ is a positive Radon measure by (6) and

$$
h u=F\left(x, u_{1}, v_{1}\right)\left(\min \left\{v_{1}, v_{2}\right\}-\varphi\right) \geq-\left|F\left(x, u_{1}, v_{1}\right)\right|\left(v_{1}-\varphi\right) \in L^{1}(\Omega) .
$$

Analogously, (12) follows from Theorem C by setting

$$
\begin{aligned}
& u=v_{2}+\left(v_{1}-v_{2}\right)^{+}-\varphi \\
& \mu=A v_{2}-F\left(x, u_{2}, v_{2}\right) \\
& h=F\left(x, u_{2}, v_{2}\right) .
\end{aligned}
$$


In this case, by monotonicity of $F$,

$$
h u= \begin{cases}F\left(x, u_{2}, v_{2}\right)\left(v_{2}-\varphi\right) & \text { in } \Omega_{1} \\ F\left(x, u_{2}, v_{2}\right)\left(v_{1}-\varphi\right) \geq F\left(x, u_{1}, v_{1}\right)\left(v_{1}-\varphi\right) & \text { in } \Omega_{2} .\end{cases}
$$

Therefore, the function $h u$ is greater than a function from $L^{1}(\Omega)$.

Now, by substituting $u=u_{i}$ and $v=v_{i}$ for $i=1,2$ in (7) and adding the obtained equalities to (11) and (12), respectively, we get

$$
\begin{aligned}
& \left\langle A v_{1},\left(v_{1}-v_{2}\right)^{+}\right\rangle \leq \int_{\Omega} F\left(x, u_{1}, v_{1}\right)\left(v_{1}-v_{2}\right)^{+} d x \\
& \left\langle A v_{2},\left(v_{1}-v_{2}\right)^{+}\right\rangle \geq \int_{\Omega} F\left(x, u_{2}, v_{2}\right)\left(v_{1}-v_{2}\right)^{+} d x
\end{aligned}
$$

which imply

$$
\left\langle A v_{1}-A v_{2},\left(v_{1}-v_{2}\right)^{+}\right\rangle \leq \int_{\Omega}\left(F\left(x, u_{1}, v_{1}\right)-F\left(x, u_{2}, v_{2}\right)\right)\left(v_{1}-v_{2}\right)^{+} d x .
$$

Since the integrated function in the right-hand side herein is equal to 0 in $\Omega_{1}$ and is non-positive in $\Omega_{2}$, we obtain from (13) that

$$
\begin{aligned}
0 \geq & \int_{\Omega}\left(\left|\nabla v_{1}\right|^{p-2} \nabla v_{1}-\left|\nabla v_{2}\right|^{p-2} \nabla v_{2}\right) \nabla\left(v_{1}-v_{2}\right)^{+} d x \\
= & \frac{1}{2} \int_{\Omega}\left(\left|\nabla v_{1}\right|^{p-2}+\left|\nabla v_{2}\right|^{p-2}\right)\left|\nabla\left(v_{1}-v_{2}\right)^{+}\right|^{2} d x \\
& +\frac{1}{2} \int_{\Omega_{2}}\left(\left|\nabla v_{1}\right|^{p-2}-\left|\nabla v_{1}\right|^{p-2}\right)\left(\left|\nabla v_{1}\right|^{2}-\left|\nabla v_{2}\right|^{2}\right) d x .
\end{aligned}
$$

Consequently, $\left(v_{1}-v_{2}\right)^{+}=0$ a.e. in $\Omega$ or, equivalently, $v_{1} \leq v_{2}$ a.e. in $\Omega$.

2) Given $u_{1}, u_{2} \in M_{0}$, set $u=\max \left\{u_{1}, u_{2}\right\}$. By monotonicity of the operator $T, u_{i} \leq T u_{i} \leq T u \quad(i=1,2)$ and so $u \leq T u$. Thus $u_{1}, u_{2} \leq u \in M_{0}$ and $M_{0}$ is directed upwards.

3) Since $T u_{0} \in K$, we can put $w=\max \left\{T u_{0}, u_{0}\right\}=u_{0}+\left(T u_{0}-u_{0}\right)^{+}$in (10) to obtain

$$
\left\langle A u_{0},\left(T u_{0}-u_{0}\right)^{+}\right\rangle \geq \int_{\Omega} F\left(x, u_{0}, u_{0}\right)\left(T u_{0}-u_{0}\right)^{+} d x .
$$

Using the same arguments that proved (11) we get

$$
\begin{aligned}
& \left\langle A T u_{0}, T u_{0}-\left(T u_{0}-u_{0}\right)^{+}-\varphi\right\rangle \\
& \quad \geq \int_{\Omega} F\left(x, u_{0}, T u_{0}\right)\left(T u_{0}-\left(T u_{0}-u_{0}\right)^{+}-\varphi\right) d x .
\end{aligned}
$$


Putting $u=u_{0}$ and $v=T u_{0}$ in (7) and adding the obtained equality to (16) we get

$$
\left\langle A T u_{0},\left(T u_{0}-u_{0}\right)^{+}\right\rangle \leq \int_{\Omega} F\left(x, u_{0}, T u_{0}\right)\left(T u_{0}-u_{0}\right)^{+} d x
$$

which together with (15) gives

$$
\begin{aligned}
& \left\langle A T u_{0}-A u_{0},\left(T u_{0}-u_{0}\right)^{+}\right\rangle \\
& \quad \leq \int_{\Omega}\left(F\left(x, u_{0}, T u_{0}\right)-F\left(x, u_{0}, u_{0}\right)\right)\left(T u_{0}-u_{0}\right)^{+} d x .
\end{aligned}
$$

Then, arguing similarly as in (13) - (14) we get $T u_{0} \leq u_{0}$ a.e. in $\Omega$. The lemma is completely proved

\section{Existence of extremal solutions}

In this section we will prove the existence of extremal solutions for inequality (1) under various conditions on the exponents $p, q, r, s$, which are given in Hypothesis (H3). To this end we shall use Theorem A to show that the operator $T$ constructed in Section 2 has extremal fixed points. Recall that we consider $T$ as an operator from $L^{p_{0}}(\Omega)$ into itself where $p_{0}=p^{*}$ or $p_{0}=q+1$.

Theorem 1. Let the function $f$ satisfy Hypotheses (H1) - (H4). Assume in addition that

(H5) $\left(p^{*}\right)^{\prime} \leq \frac{s(q-r)}{q}$.

Then inequality (1) has a maximal solution $u^{*}$ and a minimal solution $u_{*}$ in the sense that, for any solution $u$ of (1), $u_{*} \leq u \leq u^{*}$.

Proof. First, we shall use the method of Hernandez in [6] to construct a $W$-supersolution of inequality (1). By Hypothesis (H3),

$$
f(x, u)=F(x, u, 0)-(F(x, u, 0)-F(x, u, u)) \leq m(x) u^{r}-a u^{q} .
$$

It is easy to see that, for $x \in \Omega$ fixed, the function $u \mapsto m(x) u^{r}-a u^{q}$ attains its maximum $h(x)=C(m(x))^{q /(q-r)}$, where $C$ is a constant depending only on $q, r, a$. Since $m \in L^{s}(\Omega), h \in L^{t}(\Omega)$ with $t=\frac{s(q-r)}{q}$ and so $h \in L^{\left(p^{*}\right)^{\prime}}(\Omega)$ by Hypothesis (H5). Let $u_{0}=u_{1}+\|\varphi\|_{\infty}$, where $u_{1} \in W_{0}^{1, p}(\Omega)$ such that $A u_{1}=h$. Then $u_{0} \geq \varphi$, and for all $w=\max \left\{u_{0}, v\right\}=u_{0}+\left(v-u_{0}\right)^{+}$with $v \in K$ we have

$$
\left\langle A u_{0}, w-u_{0}\right\rangle=\int_{\Omega} h\left(w-u_{0}\right) d x \geq \int_{\Omega} f\left(x, u_{0}\right)\left(w-u_{0}\right) d x .
$$


Thus, $u_{0}$ is a $W$-supersolution of (1). Hence, by statement 3 ) in Lemma 2, $T u_{0} \leq u_{0}$. Since $T$ is increasing, $T\left(\left[\varphi, u_{0}\right]\right) \subset\left[\varphi, u_{0}\right]$. Therefore, if $\left\{u_{n}\right\} \subset$ $\left[\varphi, u_{0}\right]$ is an increasing sequence, then the sequence $\left\{T u_{n}\right\}$ is convergent by boundedness of the set $\left[\varphi, u_{0}\right] \subset L^{p_{0}}(\Omega)$. By Theorem A, the operator $T$ has extremal fixed points in $\left[\varphi, u_{0}\right]$, which will be the extremal solutions of inequality (1) by definition of $u_{0}$

Theorem 2. Let Hypotheses (H1) - (H3) be satisfied. Assume in addition that

(H6) $\left(p^{*}\right)^{\prime} \leq \frac{s p^{*}}{s r+p^{*}}$ and $r+1<p$.

Then inequality (1) has a maximal and a minimal solution.

Proof. First, we observe that under Hypotheses (H1) - (H3) and (H5) the operator $T$ is acting from $[\varphi, \infty) \subset L^{p^{*}}(\Omega)$ into itself. To apply Theorem A we shall prove the boundedness of the set $T\left(M_{0}\right)$ where $M_{0}=\{u: \varphi \leq u \leq T u\}$. In the sequel we will denote by $C$ a generic constant, independing on $u$. For any $v=T u \in T\left(M_{0}\right)$, from (7) we have

$$
\begin{aligned}
\langle A v, v\rangle & +\int_{\Omega}(F(x, u, 0)-F(x, u, v))(v-\varphi) d x \\
& =\int_{\Omega} F(x, u, 0)(v-\varphi) d x+\langle A v, \varphi\rangle .
\end{aligned}
$$

It follows from (2) that the left-hand side in (17) is greater than $C\left(\|v\|_{1, p}^{p}+\right.$ $\left.\|v\|_{1+q}^{1+q}-1\right)$. The first term in the right-hand side in (17) can be estimated as

$$
\begin{aligned}
\int_{\Omega} F(x, u, 0)(v-\varphi) d x & \leq \int_{\Omega} m(x) u^{r}(v-\varphi) d x \\
& \leq \int_{\Omega} m(x) v^{r}(v-\varphi) d x \\
& \leq\|m\|_{s}\left(\int_{\Omega} v^{r s^{\prime}}(v-\varphi)^{s^{\prime}} d x\right)^{1 / s^{\prime}} \\
& \leq C\left(1+\|v\|_{(1+r) s^{\prime}}^{1+r}\right) .
\end{aligned}
$$

For the term $\langle A v, \varphi\rangle$ we use the Young inequality to get

$$
\begin{aligned}
\langle A v, \varphi\rangle & =\int_{\Omega}|\nabla v|^{p-2} \nabla v \nabla \varphi d x \\
& \leq \int_{\Omega}\left(\varepsilon|\nabla v|^{(p-1) p^{\prime}}+\frac{1}{\varepsilon}|\nabla \varphi|^{p}\right) d x \\
& =\varepsilon\|v\|_{1, p}^{p}+C .
\end{aligned}
$$


Combining (17) - (19) gives

$$
\|v\|_{1, p}^{p}+\|v\|_{1+q}^{1+q} \leq C\left(1+\|v\|_{(1+r) s^{\prime}}^{1+r}\right) .
$$

Rewriting the inequality $\left(p^{*}\right)^{\prime} \leq \frac{s p^{*}}{s r+p^{*}}$ as $(1+r) s^{\prime} \leq p^{*}$ and taking into account the continuity of the imbedding $W_{0}^{1, p}(\Omega) \rightarrow L^{p^{*}}(\Omega)$, from $(20)$ we get $\|v\|_{p^{*}}^{p} \leq C\left(1+\|v\|_{p^{*}}^{1+r}\right)$ which proves the boundedness of the set $T\left(M_{0}\right)$. The theorem is proved

Theorem 3. Let Hypotheses (H1) - (H3) be satisfied. Assume in addition that

$$
\text { (H7) }\left(p^{*}\right)^{\prime} \leq \frac{s(q+1)}{s r+q+1} \text { and } r+1 \leq p .
$$

Then inequality (1) has a maximal and a minimal solution.

Proof. Under Hypotheses (H1) - (H3) and (H7), the operator $T$ acts from $[\varphi, \infty) \subset L^{q+1}(\Omega)$ into itself and we need to show the boundedeness in $L^{q+1}(\Omega)$ of the set $T\left(M_{0}\right)$ where $M_{0}=\{u: \varphi \leq u \leq T u\}$. As in the proof of Theorem 2 we have estimation (20) for all $v=T(u) \in T\left(M_{0}\right)$. If $(1+r) s^{\prime} \leq 1+q$, then (20) yields $\|v\|_{1+q}^{1+q} \leq C\left(1+\|v\|_{1+q}^{1+r}\right)$ which proves the boundedness of $T\left(M_{0}\right)$. Now, we consider the posibility $1+q<(1+r) s^{\prime}$. Rewriting the inequality $\left(p^{*}\right)^{\prime} \leq \frac{s(q+1)}{s r+q+1}$ in Hypothesis (H7) as $s^{\prime} \leq \frac{p^{*}(q+1)}{p^{*} r+q+1}$ and taking into account the monotonicity of the function $t \mapsto \frac{t}{p^{*} r+t}$ we obtain $s^{\prime} \leq \frac{p^{*}(1+r) s^{\prime}}{p^{*} r+(1+r) s^{\prime}}$ which implies $(1+r) s^{\prime}<p^{*}$. Therefore, we can apply the Gagliardo-Nirenberg inequality to get

$$
\|v\|_{(1+r) s^{\prime}} \leq C\|v\|_{1, p}^{\theta}\|v\|_{1+q}^{1-\theta}
$$

where $\theta$ is defined by $\frac{1}{1+q}-\frac{1}{(1+r) s^{\prime}}=\theta\left(\frac{1}{1+q}-\frac{1}{p^{*}}\right)$. From $(20)-(21)$ we deduce that

$$
\|v\|_{(1+r) s^{\prime}} \leq C\left(1+\|v\|_{(1+r) s^{\prime}}^{1+r}\right)^{\frac{\theta}{p}+\frac{1-\theta}{1+q}}
$$

which proves the boundedness of $T\left(M_{0}\right)$ in $L^{(1+r) s^{\prime}}(\Omega)$ since $\frac{\theta(1+r)}{p}+\frac{(1-\theta)(1+r)}{1+q}$ $\theta+(1-\theta)=1$. Therefore, the set $T\left(M_{0}\right)$ is bounded in $L^{q+1}(\Omega)$ because $1+q<(1+r) s^{\prime}$

Example. Consider variational inequality (1) with $f(x, u)=m(x) u^{r}-u^{q}$ where $r<q$ and $m \in L^{s}(\Omega)$. Functions of this type appeared in the logistic equation involving the $p$-Laplacian $[4,6,7]$

$$
\left.\begin{array}{rlrl}
-\triangle_{p} u & =\lambda m(x) u^{p-1}-u^{\gamma-1} & & \text { in } \Omega \\
u & =0 & & \text { on } \partial \Omega
\end{array}\right\}
$$


where $p<\gamma$. Hypotheses (H1) - (H3) hold with $F(x, u, v)=m(x) u^{r}-u^{q}$. According to Theorem 1, inequality (1) has extremal solutions if one of the conditions

$$
\begin{aligned}
& \left(p^{*}\right)^{\prime} \leq \min \left\{\frac{s p^{*}}{s r+p^{*}}, \frac{s(q-r)}{q}\right\} \\
& \left(p^{*}\right)^{\prime} \leq \min \left\{\frac{s(q+1)}{s r+q+1}, \frac{s(q-r)}{q}\right\}
\end{aligned}
$$

holds. These conditions are equivalent, respectively, to the conditions

$$
\begin{gathered}
s \geq \max \left\{\frac{q p^{*}}{(q-r)\left(p^{*}-1\right)}, \frac{p^{*}}{p^{*}-1-r}\right\} \quad \text { and } \quad r<p^{*}-1 \\
s \geq \max \left\{\frac{q p^{*}}{(q-r)\left(p^{*}-1\right)}, \frac{(q+1) p^{*}}{(q+1)\left(p^{*}-1\right)-r p^{*}}\right\} \quad \text { and } \quad r<\frac{(q+1)\left(p^{*}-1\right)}{p^{*}} .
\end{gathered}
$$

Analogously, by Theorems 2 and 3, problem (1) has extremal solutions if

$$
s \geq \frac{p^{*}}{p^{*}-1-r}, \quad r<p-1
$$

or

$$
s \geq \frac{(q+1) p^{*}}{(q+1)\left(p^{*}-1\right)-r p^{*}}, \quad r<\frac{(q+1)\left(p^{*}-1\right)}{p^{*}}, \quad r \leq p-1
$$

hold.

Acknowledgements. The authors are very grateful to the referees for reading the paper careful and making several corrections and remarks.

\section{References}

[1] Amann, H.: Fixed point equations and nonlinear eigenvalue problems in ordered Banach spaces. SIAM Rev. 18 (1976), 620 - 709.

[2] Boccardo, L., Giachetti, D. and F. Murat: A generalization of a theorem of $H$. Brezis and F. Browder and applications to some unilateral problems. Publ. du Lab. d'Anal. Num., Univ. Pierre et Marie Curie R89014, 1989.

[3] Carl, S. and S. Heikkila: Operator and differential equations in ordered spaces. J. Math. Anal. Appl. 234 (1999), 31 - 54.

[4] Drabek, P. and J. Hernandez: Existence and uniqueness of positive solutions for some quasilinear elliptic problems. Nonlin. Anal. 44 (2001), 189 - 204.

[5] Heikkila, S. and V. Lakshmitantham: Monotone Iterative Techniques for Discontinuous Nonlinear Differential Equations. New York - Basel: Dekker 1994.

[6] Hernandez, J.: Positive solutions for the logistic equation with unbounded weights. In: Reaction Diffusion Systems (eds.: G. Caristi and E. Mitidieri). New York: Marcel Dekker 1998, pp. 183 - 197. 
[7] Nguyen Bich Huy: Positive weak solutions for some semilinear elliptic equations. Nonlin. Anal. 48 (2002), 939 - 945.

[8] Vy Khoi Le: Existence of positive solutions of variational inequalities by a subsolution-supersolution approach. J. Math. Anal. Appl. 252 (2000), 65-90.

[9] Vy Khoi Le: Subsolution-supersolution method in variational inequalities. Nonlin. Anal. (in press).

Received 19.04.2001; in revised form 02.01.2002 